\title{
AC099850.3 Promotes Cell Proliferation and is One of Five Costimulatory Molecule-Related LncRNAs That Predict Overall Survival of Liver Hepatocellular Carcinoma
}

\author{
Meimei Liu \\ Anhui Medical College \\ Qiong Fang \\ Anhui Medical College \\ Yanping Huang \\ Anhui Medical College \\ Jin Zhou \\ Anhui Medical College \\ Qi Wang ( $\nabla$ wangqi633520@126.com ) \\ Anhui Medical College
}

\section{Research Article}

Keywords: costimulatory molecule, IncRNAs, HCC, prognostic biomarkers, carcinoma

Posted Date: November 29th, 2021

DOI: https://doi.org/10.21203/rs.3.rs-1069383/v1

License: (c) (i) This work is licensed under a Creative Commons Attribution 4.0 International License.

Read Full License 


\section{Abstract}

Background: Extensive research has revealed that costimulatory molecules play central roles in mounting anti-tumor immune responses and long non-coding RNA (IncRNA) is an important regulatory factor in the development of various cancers. However, their roles in liver hepatocellular carcinoma (HCC) remain unexplored. In this study, we aimed to explore costimulatory molecule-related IncRNAs in HCC and construct a prognostic signature to predict prognosis and improve clinical outcomes with HCC patients.

Methods: The data we used for bioinformatics analysis were downloaded from The Cancer Genome Atlas database. Costimulatory molecules were obtained from the known literature. The R software, SPSS and GraphPad Prism were used for mapping and statistical analysis.

Results: A five costimulatory molecule-related IncRNAs based risk model was initially constructed through lasso and Cox regression analysis. Moreover, multivariate regression suggested that the risk score was a significant prognostic risk factor in HCC. Samples in high-and low-risk groups exhibited significantly different in gene set enrichment analysis and immune infiltration analysis. Importantly, we found that the AC099850.3 were significantly related to cell proliferation in HCC according to the colony formation and CCK8 assays.

Conclusion: In summary, we first identified and validated a novel costimulatory molecule-related survival model and we found that AC099850.3 is closely associated with clinical stage and could remarkably facilitate cell proliferation in HCC, making it potential to be a novel therapeutic target.

\section{Introduction}

Liver cancer is one of the deadliest cancers and the third most prevalent tumor with about 905,000 new cases diagnosed and 830000 deaths worldwide in 2020(1). As one of the two histological types of liver malignant tumors, liver hepatocellular carcinoma (HCC) accounts for over $90 \%$ of all cases of liver cancer for which no targeted therapy is currently available and the mainstay of therapy remains immunotherapy and chemotherapy $(2,3)$. Despite many advances that have been made in the diagnostic modalities and standard treatments of HCC patients, the rate of recurrence and death still have remained high and the 5year overall survival rate for HCC has been anchored to only $12 \%$ during the past two decades $(4,5)$. The therapeutic effect of HCC patients largely rely on the time interval from diagnosis to the time of curative treatment initiation (TTI), especially for early-stage patients with liver cancer, so an early diagnosis and treatment is of great importance for HCC patients(6). However, most HCC patients have already progressed to the middle and late stages when they are diagnosed, thus the patients are deprived of the best treatment opportunity and have to face the poor prognosis(7). Therefore, it is critical to explore more effective targets that specifically predict prognosis and improve clinical outcomes.

As an additional treatment options for cancer patients, immunotherapy has been considered a promising therapeutic approach to surmount various cancers including $\mathrm{HCC}(8)$. In particular, immune checkpoint inhibitors (ICls), which requires a deep understanding of immunosuppressive roles in immune system 
that are generated in the tumor microenvironment (TME), has seen great success in improving clinical outcomes in multiple cancer types $(9,10)$. Studies have shown that TME system, composed of noncancer stromal cells and noncellular components, plays a key role in tumor development and progression(11). More recently, several studies are currently investigating the therapeutic potential and possible molecular mechanism of costimulatory molecules, which has great impacts on the regulation of tumor immunity in cancers $(12,13)$.

Costimulatory molecules and signals, which are composed of the B7-CD28 family and tumor necrosis factor (TNF) families, can be conceived as a new and well-regulated regulation of tumor immune microenvironment involved in various aspects of cancer biology that plays an essential role in immunotherapeutic strategies in various cancers $(14,15)$. Nowadays, a significant share of the literature focused on the potential therapeutic applications in various cancers $(15,16)$, yet there are few studies on costimulatory molecules and its biological functions in tumor immunology of HCC. Hence, we have given top priority to developing a potent and specific prognostic signature based on costimulatory molecules and signals to guide treatment decisions and improve the clinical outcomes of HCC patients.

Long noncoding RNA (IncRNA), located in the nucleus or cytoplasm, is defined as a non-coding RNA with a length of above 200 nucleotides(17). Accumulating evidences have revealed that IncRNA is associated with several stages in the gene regulation, including chromatin modification, mRNA biogenesis, and protein signaling(18). Recently, IncRNAs have been shown to own a significant effect in evaluation of cancer prognosis as well as the attractive target for therapeutic intervention in the fight against various cancers(19). For example, long noncoding RNA HULC promotes the tumorigenesis of liver cancer cells in vitro and in vivo by way of restraining PTEN via ubiquitin-proteasome system mediated by autophagyP62(20). A recent study revealed that the expression of OTUD6B-AS1 was decreased in clear cell renal cell carcinoma (ccRCC) tissues that promoted the proliferation of ccRCC cells and predicted the poorer survival in patients through the Wnt/ $\beta$-catenin signaling pathway(21). Additionally, the overexpression of H19 was significantly correlated with poor prognosis of hepatocellular carcinoma (HCC) patients, that promotes HCC invasiveness by triggering and activating the miR-193b/MAPK1 axis(22). Costimulatory molecules and signals are important regulatory pathway for tumors and other human diseases that is closely related to IncRNA(23). Therefore, it is valuable to identify key IncRNAs closely related to costimulatory molecules and prognosis in HCC. Therefore, it is very valuable to screen the costimulatory molecule IncRNAs related to the prognosis of HCC that may be useful for prognosis evaluations of HCC patients and as potential therapeutic targets for HCC.

In our study, we systematically analyzed gene expression in HCC from The Cancer Genome Atlas (TCGA) and screened out costimulatory molecule-related IncRNAs with prognostic value. Then, we constructed and validated a prognostic signature with five costimulatory molecule-related IncRNAs in the TCGA cohort. Finally, we conducted analysis on AC099850.3, the costimulatory molecule-related IncRNA we considered deserved further research in HCC.

\section{Results}




\section{Acquisition of costimulatory molecule-related IncRNAs}

The flow chart of our study was shown in Figure 1. Totally, 377 HCC samples and 50 adjacent non-tumor samples from the TCGA database were enrolled in the analysis. Moreover, 59 costimulatory molecules obtained from the known literature are listed in Table 2. Finally, a total of 132 costimulatory moleculerelated IncRNAs were identified from TCGA-HCC data through the construction of costimulatory moleculerelated mRNA and IncRNA co-expression network with the screening criteria of |Correlation Coefficient| > 0.4 and $p<0.001$ (Figure 1).

\section{Identification of costimulatory molecule-related IncRNAs with significant prognostic value in HCC}

After filtering out patients with survival time less than 30 days and with incomplete clinical data, a total of $343 \mathrm{HCC}$ patient samples were randomly assigned into training cohort $(n=172)$ and testing cohort $(n$ $=171$ ) and were shown in Table 1 . Then we merged these data with costimulatory molecule-related IncRNAs to obtain clinical information and costimulatory molecule-related IncRNAs expression of HCC patients. According to univariate Cox regression proportional hazards analysis, 36 costimulatory molecule-related IncRNAs which related to prognosis have been selected (Table S1), including 2 IncRNAs with low risk (hazard ration $(H R)<1$ ) and 34 IncRNAs with high risk (hazard ration $(H R)>1$ ), and 22 IncRNAs with the lowest $p$ value were shown in Figure 2A. Furthermore, 31 OS-related IncRNAs were extracted after 1,000 iterations using LASSO Cox regression analysis and multivariate Cox proportional hazards regression analysis (Figure 2C,D). Ultimately, as shown in Figure 2B, we constructed a costimulatory molecule-related IncRNA risk prognostic signature consisting of 5 OS-related costimulatory molecule-related IncRNAs including BOK-AS1, AC099850.3, AL365203.2, NRAV and AL049840.4 and the coefficients of each costimulatory molecule-related IncRNAs were obtained from the model (Table S2). Then, we analyzed the expression of these prognostic IncRNAs between HCC samples and adjacent nontumor samples (Figure 2E) and Kaplan-Meier (K-M) survival analysis was performed to compare the OS time between the high expression group and low expression group with each prognostic IncRNA (Figure 2F-J).

\section{Evaluation and verification of the prognostic signature containing 5 OS-related IncRNAs}

On the basis of the riskscore formula and the calculated median risk score, patients in training and testing cohort were both classified into high-risk score group and low-risk score group. Kaplan-Meier survival analysis revealed that the OS time of the high-risk score group for HCC patients was significantly shorter than the low-risk score group both in training cohort (Figure 3A) and testing cohort (Figure 3D), indicating that risk score could predict the prognosis (all $p<0.001$ ). The distributions of the risk score and the relevant survival statuses for each patient were visualized by risk curve and scatterplot, which demonstrated that mortality occurrence of patients depended on the riskscore (Figure 3B,E). The heatmap of these 5 OS-related IncRNAs expressions in HCC samples showed that NVAR, AC099850.3, AL3652.3.2 
and AL049840.4 seemed to be risk factors and all were highly expressed in the high-risk group, while BOK-AS1 seemed to be a protective factor that was up-regulated in the low-risk group both in training cohort (Figure 3B) and testing cohort (Figure 3E). In addition, univariate and multivariate Cox regression analyses were performed to determine whether the five costimulatory molecule-Related IncRNAs could be used as the independent prognosis biomarkers of patients in HCC. Univariate Cox regression showed that stage $(p<0.001,95 \% \mathrm{Cl} 1.612-2.723), T(p<0.001,95 \% \mathrm{Cl} 1.575-2.554)$ and riskscore $(p<0.001,95 \% \mathrm{Cl}$ 1.234-1.503) were related to the prognosis and only the risk score $(p<0.001,95 \% \mathrm{Cl} 1.179-1.476)$ was associated with prognosis in multivariate Cox regression analysis respectively, suggesting that the risk model of the five costimulatory molecule-Related IncRNAs is an independent prognostic factor for patients with HCC (Figure 3G,H). Furthermore, the area under the receiver operating characteristic (ROC) curve (AUC) of the risk score was calculated to assess the predictive sensitivity and specificity of the riskscore on the prognosis with HCC patients. The AUC value of the risk score was 0.704 in training cohort and 0.786 in testing cohort (Figure 3C,F), indicating that these 5 costimulatory molecule-related IncRNAs were quite reliable for the prognostic risk model of HCC. Taken together, these data all demonstrate that the prognostic signature of the five costimulatory molecule-related IncRNAs is a significant independent prognostic factor and is of great robust utility for predicting the prognosis in HCC patients.

\section{Gene set enrichment analysis in two riskscore group}

Gene Set Enrichment Analysis (GSEA) was applied between the high-risk score group and low-risk score group to identify the potential enhanced signaling pathway. The top 8 hallmark functions of the biomarker gene sets in the high-risk score and low-risk score groups based on GSEA are shown in Figure 4A and Figure 4D. In addition, Figure 4B revelved that the top 5 kegg pathways were significantly enriched in the high-risk group including hematopoietic cell lineage, DNA replication, primary immunodeficiency, cell cycle, ECM receptor interaction, and Figure 4E showed that the top 5 kegg pathways in the low-risk group were complement and coagulation cascades, drug metabolism cytochrome p450, fatty acid metabolism, metabolism of xenobiotics by cytochrome p450 and peroxisome. The top 10 altered gene sets in GO pathways between the high-risk and low-risk groups are shown in Figure 4C and Figure 4F and the result suggested that the high-risk score group correlates with cancer, whereas the low-risk score group correlates with enhanced oxidation.

\section{Immunity analysis of HCC in two riskscore group}

To further elucidate the effects of high and low expression levels on immune cell infiltration type as well as its related immune function between the two risk stratifications, TIMER 2.0 was used to quantify immune infiltration based on the costimulatory molecule-related IncRNA signature. As shown in Figure $5 A$, the proportions of tumor-infiltrating CD8 + T cell was significantly lower and the expression of M0 Macrophage was higher in high-risk patients. Correlation analysis between immune cell subpopulations and related functions revealed that T cell functions (including Tfh, Treg, type I IFN response and type II IFN response) and aDCs, iDCs and CCR were significantly lower in the high- risk group than in the low-risk group (Figure 5B). These observations suggested that the infiltration of these immune cell types as well 
as its related immune function might exert an important influence on the prognosis of HCC patients. Given the expanding role of therapeutic strategies based on immune checkpoint blockade in HCC, we then made use of the online tool Tumor Immune Dysfunction and Exclusion (TIDE, http://tide.dfci.harvard.edu) to explore the association between the risk stratifications and the effect of immune checkpoint inhibition therapy (Figure 5C-E).

\section{Clinical correlation analysis and the immunity features of AC099850.3}

To further investigate whether the 5 costimulatory molecule-related IncRNAs participated in the development of HCC, we determined the relevance between the expression of the 5 costimulatory molecule-related IncRNAs with clinicopathological parameters. There was significant association between the costimulatory molecule-related IncRNA AC099850.3 and clinicopathological factors with HCC, including grade, AJCC stage, T and M stage, as shown in Figure 6C-F. What's more, the heatmap and lollipop-diagram showed that AC099850.3 have a strong correlation with ICls and T cell functions including Th2 cells and Thelper cells (Figure 6A,B).

\section{AC099850.3 promotes HCC cell proliferation in vitro}

\section{AC099850.3 expression was upregulated in HCC tissues and cell lines}

Based on five paired HCC tissue, the results of qPCR showed that the expression of AC099850.3 was prominent upregulation in tumoral tissues ( $p<0.01$, Figure $7 A$ ), which was also validated in cell level (Figure 7B). Then, Hep3B and SUN-449 were transfected with target sequence against AC010973.2 for further research due to their highest expression level of AC099850.3 and the knockdown of AC099850.3 was verified by mRNA expression detection (Figure 7C).

\section{AC010973.2 promoted tumor proliferation in HCC}

The results of colony formation assays revealed that clonogenic survival was significantly decreased in si-AC099850.3 group than the control group in both Hep3B and SUN-449 cell lines (Figure 7D), suggesting that AC099850.3 knockdown weakened the ability of tumor cells. Similar results were demonstrated in the CCK8 assays, which showed that the knockdown of AC099850.3 prominently inhibited the proliferation capacity of HCC cell lines (Figure 7E,F). Altogether, these results indicated that AC099850.3 expression promotes tumor growth and survival take in $\mathrm{HCC}$, presumably by favoring cancer cell survival in a tissue environment.

\section{Discussion}

Liver hepatocellular carcinoma (HCC) accounts for approximately $90 \%$ of all liver cancer, one of the most frequent malignancies globally with a high probability of metastasis and recurrence(2). Due to most HCC patients are diagnosed at the middle and late stages when surgical resection is not an option, 
immunotherapy and systemic therapies remain optimal for treatment of HCC patients which brings the patients great suffering and heavy financial burden as well as a worse prognosis(24). Therefore, it raises an urgent need for the development of sensitive and reliable prognostic biomarkers to identify patients with poor prognosis and who can benefit from early adjuvant treatment instead of salvage treatment. In recent years, there has been an increasing number of researches focused on molecular characteristic in early diagnosis and prognosis improvement. Significantly, it was generally believed that some functional IncRNAs may be instrumental for researchers to better unravel the initial process of malignant cancer progression and develop more efficient measures to improve the prognosis for patients(25). Most notably, some costimulatory molecule-related signatures have been revealed to be highly associated with clinical features and stratify patients into two subgroups with different prognoses which can help guide treatment for patients(26). However, there is little share of the literature focused on the potential therapeutic and prognostic role of costimulatory molecule-related IncRNAs in HCC.

In this study, we identified a novel costimulatory molecule-related five-IncRNAs signature in a TCGA cohort using the Cox regression model, including a training dataset and a testing dataset, demonstrating its sensitivity and specificity. The multivariate Cox regression analysis indicated that only the riskscore of our signature was an appropriate independent predictive factor for HCC patients and was significantly correlated with prognosis in different clinicopathological parameters. Further study suggested that our prognostic signature was associated the tumor immune microenvironment and immunotherapy response, which might provide valuable information for predicting the prognosis of HCC patients and help guide physicians find the optimal immunotherapy for patients. Additionally, the results of colony formation assays showed that the knockdown of AC099850.3 remarkably inhibited the proliferation capacity of HCC cell lines.

Recently, studies showed that the costimulatory molecules play an important role in the regulation of tumor immunity. For instance, the discover of immune checkpoints in B7-CD28 family, one of the two main costimulatory molecule families, has opened new possibilities to induce durable tumor regressions using monoclonal antibodies $(\mathrm{mAb})(27)$. Kanodia et al. reported that overexpression of costimulatory molecule TNFSF14 could contribute to the expansion of tumor antigen-specific T-cells to prevent the growth of human papillomavirus 16-induced tumors by altering the tumor microenvironment(28). Moreover, in patients with HBV-related liver cancer, the expression of PD-1/PD-L1 was upregulated in tumor-infiltrating lymphocytes (TIL) which contributes to the portal vein thrombosis, indicating that the potential of PD-1 expression in TIL of liver tissues may function as a prognostic marker. In the meantime, it hasn't escaped our notice that more and more IncRNAs were proven to be related to the development and progression of liver cancer and were employed to the diagnosis, prognosis, and therapy. For example, the long noncoding RNA IncTCF7 was found to express highly in HCC tumors and was authenticated that can promote liver cancer stem cells (CSCs) self-renewal and tumor progress by activating of Wnt signaling(29). A previous clinical investigation revealed that the expression of Inc-DILC in patient HCCs was reduced and demonstrated the correlation with the expression level of IL-6 and CD24, suggesting that Inc-DILC could be not only a potential prognostic biomarker, but also a promising therapeutic target

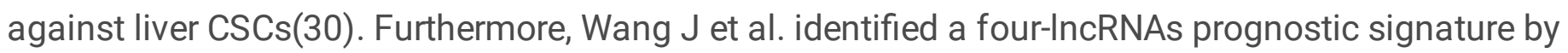


comprehensive bioinformatics analysis in the liver cancer patients, which can specifically predict prognosis for liver cancer and improve clinical outcomes(31).

Given the crucial role of IncRNAs in the malignant progression of HCC and the relevance with costimulatory molecules, gene activation, metabolism, the immune response and other biological processes. We identified 132 costimulatory molecule-related IncRNAs from TCGA-HCC through the construction of related mRNA and IncRNA co-expression network. After using lasso and Cox proportional hazards regression analyses, five costimulatory molecule-related IncRNAs (BOK-AS1, AC099850.3, AL365203.2, NRAV, and AL049840.4) with prognostic values were selected. Among these 5 IncRNAs, AC099850.3, AL365203.2, NRAV, and AL049840.4 were risk factors upregulated in high-risk score group, whereas BOK-AS1 was protective factors, which were downregulated in high-risk score group. AC099850.3 was upregulated in HCC patients, which could promote the migration and proliferation of HCC, suggesting the high expression of IncRNA AC099850.3 represents a poor prognosis of HCC patients(32). A recent study showed that IncRNA AL365203.2 and NRAV are associated with a poor prognosis and regulate the infiltration of numerous immune cell types as well as tumor progression in $\mathrm{HCC}(33)$. The functions of BOK-AS1 and AL049840.4 in HCC remain unclear. Above all, these costimulatory molecular-related IncRNAs were novel and were needed to be investigated in HCC.

In our research, GSEA analysis showed that cancer-related pathways were significantly enriched in the high-risk group, including epithelial mesenchymal transitions, which is involved in multiple signal transduction pathways and is closely related to the invasion and metastasis of tumor cells(34). Moreover, cell cycle can not only protect tumor cells from different stresses but also promote tumor progression and the $B$ cell receptor signaling pathway can ultimately activate the expression of genes involved in B cell proliferation, differentiation as well as other tumor processes(35). This suggests that the five costimulatory molecular-related IncRNAs we have identified are related to the occurrence and development of HCC. To further explore the associations between our signature and tumor immune microenvironment, the immune cell infiltration type as well as its related immune function between the two risk stratifications were compared. The results showed that the tumor-infiltrating CD8 $+\mathrm{T}$ cell and $T$ cell functions (including Tfh, Treg, type I IFN response and type II IFN response) and aDCs, iDCs and CCR were significantly lower in the high- risk group while the expression of MO Macrophage and NK cell were higher in high-risk patients. What's more, research revealed that the percentages of exhausted CD8+ T cell were increased significantly in liver cancer samples, respectively, the patients at the late stage have a higher exhaustion level than other patients which confirmed its association with poor prognosis in liver cancer(36). Larger macrophages were more frequently found in colorectal liver metastasis patients with worse prognosis(37). Furthermore, there is accumulating evidence that the infiltration of a large number of Treg cells is often associated with poor prognosis which brings the current cancer immunotherapy more difficulties and other uncertain factors(38). The TIDE score is a newly-developed computational method to model tumor immune evasion, and considered a more accurate biomarker than TMB or PD-L1 expression. Collectively, high-risk patients exhibited high Exclusion score and the score of Dysfunction and TIDE with high-risk patients was obvious low, implying that high-risk patients may be suitable for immunotherapy. AC099850.3, as the most critical model IncRNA, was selected for further research. 
Results of the clinical relevance between the expression of the IncRNA AC099850.3 and clinicopathological parameters showed that AC099850.3 highly expressed in later stage of HCC,

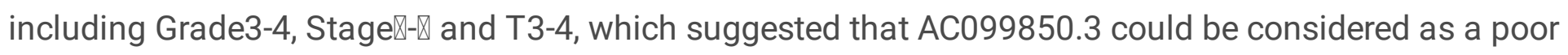
prognostic marker. To validate whether AC099850.3 have function in promoting the development of HCC, qPCR was performed and results showed that the expression of AC099850.3 IncRNA in tumor tissues was significantly up-regulated. Besides, colony formation assays and CCK8 analysis also showed that the knockdown AC099850.3 could significantly inhibit the proliferation of HCC cell lines.

On the whole, the strength of our study was that we successfully constructed and tested a costimulatory molecule-related prognostic signature for HCC, which could be used to stratify patients to accurately predict the prognostic outcomes with HCC, and AC099850.3 could meaningfully promote cell proliferation of HCC. However, this study was bound by some limitations. First of all, our signature should be further validated in not only TCGA data but in other prospective cohorts. Secondly, 5 IncRNAs related to costimulatory molecule, especially, AC099850.3 need to be further studied, including functional experiments and potential molecular mechanisms.

\section{Materials And Methods}

\section{Data preparation and sources}

All cases of liver cancer in this study were exclusively hepatocellular carcinoma (HCC). The data of patients with HCC including RNA sequencing normalized as FPKM and clinical information of the HCC patients were downloaded from The Cancer Genome Atlas (TCGA) database (https://portal.gdc.cancer.gov/), which included $377 \mathrm{HCC}$ tissues and 50 normal tissues. Costimulatory molecules, which were related to HCC, were collected from the known literature(39). All the costimulatory molecules were listed in Supplementary Table 2.

\section{Screening of costimulatory molecule-related IncRNAs in HCC}

We first identified 59 costimulatory molecules from the known literature. Then, pearson correlation analysis was adopted to determine the correlation between the expression of the IncRNAs and the corresponding costimulatory molecules by "limma" R package(40). The IncRNAs we regarded as being related to costimulatory molecule based on the criteria that |Correlation Coefficient $\mid>0.4$ and the $P$ value was less than $0.001(p<0.001)$.

\section{Construction of costimulatory molecule-related IncRNA prognostic signature}

After excluding patients with missing clinical information and survival time less than 30 days to eliminate non-cancer related deaths, a total of $343 \mathrm{HCC}$ samples were randomly assigned into training cohort $(\mathrm{n}=$ 172) and testing cohort $(n=171)$, and the detailed clinicopathological parameters of patients with HCC, 
including age, gender, stage, TNM stage, grade and cancer status of HCC were shown in Supplementary Table 1. We applied the training cohort to construct a prognostic signature and evaluated it in the testing cohort. Univariate Cox regression analysis was assessed to obtain the costimulatory molecule-related IncRNAs that were associated with the overall survival (OS) of HCC patients in the training group according to the criteria of $p<0.05$. Then we used lasso regression analysis by the "glmnet" $R$ package with the optimal value of penalty parameter $(\lambda)$ determined according to the tenfold cross-validations that used to select significant features(41). Finally, multivariate Cox analysis was performed to construct a prognostic model and the IncRNAs with independent prognostic predictive value were enrolled for the construction of the riskscore model with the following formula:

$$
\text { riskscore }=\sum_{i=1}^{k} \operatorname{coef}(\operatorname{lncRNAi}) * \exp (\operatorname{lncRNAi})
$$

In this formula, $\exp (\operatorname{IncRNAi})$ indicated the expression of IncRNA and coef (IncRNAi) is the correlation coefficient of the IncRNA in the riskscore model.

\section{Evaluation and verification of the prognostic signature}

The above formula was used to calculate the risk score for each HCC patient and the patients were divided into high-risk score group and low-risk score group based on their prognostic riskscore.

Kaplan-Meier (K-M) survival analysis was performed to compare the overall survival (OS) time between the high- and low-risk groups. The receiver operating characteristics (ROC) and the area under the ROC (AUC) were plotted to estimate the predictive accuracy of the model by "survivalROC" R package. Furthermore, distribution curves, scatter dot plot, and heatmap were utilized to visualized the riskscore distribution, number of censored patients and prognosis-related IncRNAs in two groups. Univariate and multivariate Cox regression analyses between the riskscore and different clinical factors (including age, gender, grade, stage, $T, M$, and $N$ ) was performed to assess whether the riskscore could be used as an independent indicator for the prognosis of HCC using the "survival" R package.

\section{Gene set enrichment analysis}

To further explore the pathways and biological processes that prognostic IncRNAs may participate in. Gene set enrichment analysis (GSEA) was performed to the two risk stratifications of the molecule-related IncRNA prognosis signature based on the "h.all.v7.4.symbols.gmt", "c5.all.v7.2.symbols.gmt" and "c2.cp.kegg.v7.4.symbols.gmt" reference package in GSEA software.

\section{Exploration of immune features based on prognostic signature}

We obtained immune cell data from the Tumor Immune Estimation Resource (TIMER) database to determine the relationship between the immune cell infiltration type as well as its related immune function and riskscore(42). Then we investigated the densities of 22 immune cell types with tumor and 
calculated the activity of 29 immune-related function between high-risk score group and low-risk score group. The transcriptome profiles from TCGA datasets were analyzed through the online tool Tumor Immune Dysfunction and Exclusion (TIDE, http://tide.dfci.harvard.edu) to predict the immunotherapeutic responses of each sample.

\section{Analysis of correlation between prognostic IncRNAs and clinicopathological parameters}

Among the five IncRNAs, AC099850.3 was primarily expressed in HCC tissues and it was the most significant one with the lowest $\mathrm{p}$ value for OS through multivariate Cox analysis. Therefore, to assess the significance of AC099850.3 on the correlation with clinicopathological parameters, violin plots were performed to visualize the differential expression in clinicopathological factors, respectively. Furthermore, heatmap and lollipop-diagram were used to show the correlation between immune functions and AC099850.3.

\section{Expression of the AC099850.3 in cell lines of HCC in vitro RTqPCR assay}

Human HCC cell lines (HepG2, Hep3B, BEL-7402 and SUM-449) and normal hepatic cell line LO2 purchased from Cell Bank of Chinese Academy of Sciences (Shanghai, China) were used for qRT-PCR. Total cellular RNA was extracted using an RNA extraction kit according to the manufacturer's protocol. The cDNA synthesis was reverse-transcribed using a reverse transcription kit (TaqMan). The qPCR assay then was conducted with SYBR Green methods. The sequences of all primers used in this study were as followed: AC099850.3, forward: 5'-CTGGAGTGGCAGTGTTGCAATC-3'; AC099850.3, reverse: 5'GGTGACGCACACCTGTAGTCC-3'; GAPDH, forward: 5'-GAAGGTGAAGGTCGGAGTC-3'; GAPDH, reverse: 5'GAAGATGGTGAT GGGATTTC-3'.

\section{RNA interference}

RNA interference was performed with lipofectamine 2000 transfection reagent. The target sequence used for siRNA against AC010973.2 was: siRNA1, 5'-TGGGTAATTTTAAATTTTTTGA-3'; siRNA2, 5'CTGTATTACTGTCTTTGATTA-3'; siRNA3, 5'-TTGTCATAAAATTTTTATGTA-3'.

\section{Colony Forming Assay}

The Hep3B and SUN-449 cells were transfected with IncRNA-targeted siRNAs for clonogenic assay. Afterward, 500 cells were plated in six-well plates and incubated for a minimum of 14 days. The cellular colonies were counted by staining with crystal violet.

\section{Cell proliferation assay}

CCK-8 (Dojindo, Shanghai, China) assay was performed for the evaluation of cell proliferation ability. In brief, cells were plated into 96-well plates with 2000 cell per well and then cultured at $37^{\circ} \mathrm{C}$ for $24 \mathrm{~h}, 48 \mathrm{~h}$ 
and $72 \mathrm{~h}$. After adding 10\% CCK8 reagent into the corresponding wells, the absorbance value at $450 \mathrm{~nm}$ was measured to express the cell proliferation ability.

\section{Conclusion}

In conclusion, we identified and validated a novel costimulatory molecule-related survival model consisting of 5 IncRNAs (BOK-AS1, AC099850.3, AL365203.2, NRAV, and AL049840.4) and demonstrated AC099850.3 could promote the proliferation of HCC. In the future, these 5 costimulatory molecule-related may become more effective targets for the treatment of HCC and provide a still further personalized and accurate prognostic surveying tool.

\section{Declarations}

\section{Acknowledgments}

The authors thank the Center Lab of Anhui Medical College for the selfless helps and supports on their experimental technology and experimental.

\section{Declaration of Conflicting Interests}

The authors declare no competing interests.

\section{Author Contributions}

LM and FQ collected the data and performed all analysis. HY and ZJ wrote the manuscript. All the authors participated in the data analysis and approved the final version of the manuscript.

\section{Ethical Statement}

Not applicable.

\section{Funding}

The author(s) received no financial support for the research, authorship, and/or publication of this article.

\section{Supplemental Material}

Supplementary material for this article is available online

\section{Reference}

1. Sung H, Ferlay J, Siegel R, Laversanne M, Soerjomataram I, Jemal A, et al. Global Cancer Statistics 2020: GLOBOCAN Estimates of Incidence and Mortality Worldwide for 36 Cancers in 185 Countries. CA: a cancer journal for clinicians. 2021;71(3):209-49. 
2. Anwanwan D, Singh S, Singh S, Saikam V, Singh R. Challenges in liver cancer and possible treatment approaches. Biochimica et biophysica acta Reviews on cancer. 2020;1873(1):188314.

3. Sia D, Villanueva A, Friedman S, Llovet J. Liver Cancer Cell of Origin, Molecular Class, and Effects on Patient Prognosis. Gastroenterology. 2017;152(4):745-61.

4. Iwagami Y, Huang CK, Olsen MJ, Thomas JM, Jang G, Kim M, et al. Aspartate $\beta$-hydroxylase modulates cellular senescence through glycogen synthase kinase $3 \beta$ in hepatocellular carcinoma. Hepatology. 2016.

5. Petrowsky H, Fritsch R, Guckenberger M, De Oliveira M, Dutkowski P, Clavien P. Modern therapeutic approaches for the treatment of malignant liver tumours. Nature reviews Gastroenterology \& hepatology. 2020;17(12):755-72.

6. Bai L, Liu Z, Fang Q, Yan Q, Shi O, Bao P, et al. The trends and projections in the incidence and mortality of liver cancer in urban Shanghai: a population-based study from 1973 to 2020. Clinical epidemiology. 2018;10:277-88.

7. Kim D, Talati C, Kim R. Hepatocellular carcinoma (HCC): beyond sorafenib-chemotherapy. Journal of gastrointestinal oncology. 2017;8(2):256-65.

8. Kim OY, Park HT, Dinh NTH, Choi SJ, Lee J, Kim JH, et al. Bacterial outer membrane vesicles suppress tumor by interferon-y-mediated antitumor response. Nat Commun. 2017;8(1):626-.

9. Hollern DP, Xu N, Thennavan A, Glodowski C, Garcia-Recio S, Mott KR, et al. B Cells and T Follicular Helper Cells Mediate Response to Checkpoint Inhibitors in High Mutation Burden Mouse Models of Breast Cancer. Cell. 2019;179(5):1191-206.e21.

10. Singh S, Hassan D, Aldawsari HM, Molugulu N, Shukla R, Kesharwani P. Immune checkpoint inhibitors: a promising anticancer therapy. Drug discovery today. 2020;25(1):223-9.

11. Zhang M, Di Martino JS, Bowman RL, Campbell NR, Baksh SC, Simon-Vermot T, et al. AdipocyteDerived Lipids Mediate Melanoma Progression via FATP Proteins. Cancer Discov. 2018;8(8):1006-25.

12. Wei SC, Duffy CR, Allison JP. Fundamental Mechanisms of Immune Checkpoint Blockade Therapy. Cancer Discov. 2018;8(9):1069-86.

13. Schildberg F, Klein S, Freeman G, Sharpe A. Coinhibitory Pathways in the B7-CD28 Ligand-Receptor Family. Immunity. 2016;44(5):955-72.

14. Croft M, Benedict C, Ware C. Clinical targeting of the TNF and TNFR superfamilies. Nature reviews Drug discovery. 2013;12(2):147-68.

15. Aye L, Song X, Yang J, Hu L, Sun X, Zhou J, et al. Identification of a Costimulatory Molecule Gene Signature to Predict Survival and Immunotherapy Response in Head and Neck Squamous Cell Carcinoma. Frontiers in cell and developmental biology. 2021;9:695533-.

16. Ge Y, Chen W, Zhang X, Wang H, Cui J, Liu Y, et al. Nuclear-localized costimulatory molecule 4-1BBL promotes colon cancer cell proliferation and migration by regulating nuclear Gsk3 $\beta$, and is linked to the poor outcomes associated with colon cancer. Cell Cycle. 2020;19(5):577-91. 
17. Cai $P$, Otten A, Cheng $B$, Ishii $M$, Zhang $W$, Huang $B$, et al. PRANCRA genome-wide long noncoding RNA CRISPRi screen identifies as a novel regulator of epidermal homeostasis. Genome research. 2020;30(1):22-34.

18. Satpathy A, Chang $\mathrm{H}$. Long noncoding RNA in hematopoiesis and immunity. Immunity. 2015;42(5):792-804.

19. Schmitt AM, Chang HY. Long Noncoding RNAs in Cancer Pathways. Cancer Cell. 2016;29(4):452-63.

20. Xin X, Wu M, Meng Q, Wang C, Lu Y, Yang Y, et al. Long noncoding RNA HULC accelerates liver cancer by inhibiting PTEN via autophagy cooperation to miR15a. Molecular cancer. 2018;17(1):94.

21. Wang G, Zhang Z, Jian W, Liu P, Xue W, Wang T, et al. Novel long noncoding RNA OTUD6B-AS1 indicates poor prognosis and inhibits clear cell renal cell carcinoma proliferation via the $\mathrm{Wnt} / \beta$ catenin signaling pathway. Molecular cancer. 2019;18(1):15.

22. Ye Y, Guo J, Xiao P, Ning J, Zhang R, Liu P, et al. Macrophages-induced long noncoding RNA H19 upregulation triggers and activates the miR-193b/MAPK1 axis and promotes cell aggressiveness in hepatocellular carcinoma. Cancer letters. 2020;469:310-22.

23. Yang C, Li J, Yen J, Lai I, Ho Y, Chen Y, et al. IncRNA NTT/PBOV1 Axis Promotes Monocyte Differentiation and Is Elevated in Rheumatoid Arthritis. International journal of molecular sciences. 2018;19(9).

24. Lee J, Hwang J, Chun H, Woo W, Oh S, Choi J, et al. PLEKHA8P1 Promotes Tumor Progression and Indicates Poor Prognosis of Liver Cancer. International journal of molecular sciences. 2021;22(14).

25. Dickson I. Hepatocellular carcinoma: A role for IncRNA in liver cancer. Nature reviews Gastroenterology \& hepatology. 2016;13(3):122-3.

26. Zhang C, Zhang Z, Sun N, Zhang Z, Zhang G, Wang F, et al. Identification of a costimulatory molecule-based signature for predicting prognosis risk and immunotherapy response in patients with lung adenocarcinoma. Oncoimmunology. 2020;9(1):1824641.

27. Podlesnykh SV, Abramova KE, Gordeeva A, Khlebnikov Al, Chapoval Al. Peptide Blocking CTLA-4 and B7-1 Interaction. Molecules. 2021;26(2):253.

28. Kanodia S, Da Silva D, Karamanukyan T, Bogaert L, Fu Y, Kast W. Expression of LIGHT/TNFSF14 combined with vaccination against human papillomavirus Type 16 E7 induces significant tumor regression. Cancer research. 2010;70(10):3955-64.

29. Wang Y, He L, Du Y, Zhu P, Huang G, Luo J, et al. The long noncoding RNA IncTCF7 promotes selfrenewal of human liver cancer stem cells through activation of Wnt signaling. Cell stem cell. 2015;16(4):413-25.

30. Wang X, Sun W, Shen W, Xia M, Chen C, Xiang D, et al. Long non-coding RNA DILC regulates liver cancer stem cells via IL-6/STAT3 axis. Journal of hepatology. 2016;64(6):1283-94.

31. Wang J, Pu J, Yao T, Lu X, Deng Y. Four long noncoding RNAs as potential prognostic biomarkers for hepatocellular carcinoma. Journal of cellular physiology. 2019;234(6):8709-16. 
32. Wu F, Wei H, Liu G, Zhang Y. Bioinformatics Profiling of Five Immune-Related IncRNAs for a Prognostic Model of Hepatocellular Carcinoma. Frontiers in oncology. 2021;11:667904.

33. Zhou P, Lu Y, Zhang Y, Wang L. Construction of an Immune-Related Six-IncRNA Signature to Predict the Outcomes, Immune Cell Infiltration, and Immunotherapy Response in Patients With Hepatocellular Carcinoma. Frontiers in oncology. 2021;11:661758.

34. Dongre A, Weinberg R. New insights into the mechanisms of epithelial-mesenchymal transition and implications for cancer. Nature reviews Molecular cell biology. 2019;20(2):69-84.

35. Petroni G, Formenti S, Chen-Kiang S, Galluzzi L. Immunomodulation by anticancer cell cycle inhibitors. Nature reviews Immunology. 2020;20(11):669-79.

36. Zheng C, Zheng L, Yoo JK, Guo H, Zhang Y, Guo X, et al. Landscape of Infiltrating T Cells in Liver Cancer Revealed by Single-Cell Sequencing. Cell. 2017;169(7):1342-56.e16.

37. Donadon M, Torzilli G, Cortese N, Soldani C, Di Tommaso L, Franceschini B, et al. Macrophage morphology correlates with single-cell diversity and prognosis in colorectal liver metastasis. J Exp Med. 2020;217(11):e20191847.

38. Tanaka A, Sakaguchi S. Regulatory T cells in cancer immunotherapy. Cell research. 2017;27(1):10918.

39. Aye L, Song X, Yang J, Hu L, Sun X, Zhou J, et al. Identification of a Costimulatory Molecule Gene Signature to Predict Survival and Immunotherapy Response in Head and Neck Squamous Cell Carcinoma. Frontiers in cell and developmental biology. 2021;9:695533.

40. Ritchie M, Phipson B, Wu D, Hu Y, Law C, Shi W, et al. limma powers differential expression analyses for RNA-sequencing and microarray studies. Nucleic acids research. 2015;43(7):e47.

41. Engebretsen S, Bohlin J. Statistical predictions with glmnet. Clinical epigenetics. 2019;11(1):123.

42. Li T, Fu J, Zeng Z, Cohen D, Li J, Chen Q, et al. TIMER2.0 for analysis of tumor-infiltrating immune cells. Nucleic acids research. 2020;48:W509-W14.

\section{Tables}

Table 1 Patients' clinical features of training set and testing set. 
Covariate

Total Training set Testing set

\begin{tabular}{|c|c|c|c|c|}
\hline & & & \\
\hline & & $N=343$ & $\mathrm{~N}=172$ & $N=171$ \\
\hline \multirow[t]{2}{*}{ Age (years), no (\%) } & $\leq 65$ & $216(63.0)$ & $55(32.0)$ & $99(57.9)$ \\
\hline & $\square 65$ & $127(37.0)$ & $117(68.0)$ & $72(42.1)$ \\
\hline \multirow[t]{2}{*}{ Gender (years), no (\%) } & Female & $110(32.1)$ & $53(30.8)$ & $57(33.3)$ \\
\hline & Male & $233(67.9)$ & $119(69.2)$ & $114(66.7)$ \\
\hline \multirow[t]{2}{*}{ Vital status, no (\%) } & Alive & $220(64.1)$ & $110(64.0)$ & $110(64.3)$ \\
\hline & Dead & $123(35.9)$ & $62(36.0)$ & $61(35.7)$ \\
\hline \multirow[t]{5}{*}{ Grade, no (\%) } & G1 & $53(15.5)$ & $22(12.8)$ & $31(18.1)$ \\
\hline & G2 & $161(46.9)$ & $85(49.4)$ & $76(44.4)$ \\
\hline & G3 & $112(32.7)$ & $55(32.0)$ & $57(33.3)$ \\
\hline & G4 & $12(3.5)$ & $8(4.7)$ & $4(2.3)$ \\
\hline & unknow & $5(1.5)$ & $2(1.2)$ & $3(1.8)$ \\
\hline \multirow[t]{3}{*}{ Stage, no (\%) } & Stage I-II & $238(69.4)$ & $115(66.9)$ & $123(71.9)$ \\
\hline & Stage III-IV & $83(24.2)$ & $43(25.0)$ & $40(23.4)$ \\
\hline & unknow & $22(6.4)$ & $14(8.1)$ & $8(4.7)$ \\
\hline \multirow[t]{5}{*}{ T state, no (\%) } & $\mathrm{T} 1$ & $168(49)$ & $84(48.8)$ & $84(49.1)$ \\
\hline & $\mathrm{T} 2$ & $84(24.5)$ & $40(23.3)$ & $44(25.7)$ \\
\hline & T3 & 75 (21.9) & $39(22.7)$ & $36(21.1)$ \\
\hline & T4 & $13(3.8)$ & $8(4.7)$ & $5(2.9)$ \\
\hline & TX & $3(0.9)$ & $1(0.6)$ & $2(1.2)$ \\
\hline \multirow[t]{3}{*}{ N stage, no (\%) } & NO & $239(69.7)$ & $119(69.2)$ & $120(70.1)$ \\
\hline & N1 & $3(0.9)$ & $1(0.6)$ & $2(1.2)$ \\
\hline & NX & $101(29.4)$ & $52(30.2)$ & $49(28.7)$ \\
\hline \multirow[t]{3}{*}{ M stage, no (\%) } & MO & 245 (71.4) & $122(70.9)$ & 123 (71.9) \\
\hline & M1 & $3(0.9)$ & $2(1.2)$ & $1(0.6)$ \\
\hline & $M X$ & 95 (27.7) & 48 (27.9) & $47(27.5)$ \\
\hline
\end{tabular}

Due to technical limitations, table 2 is only available as a download in the Supplemental Files section. 
Figures

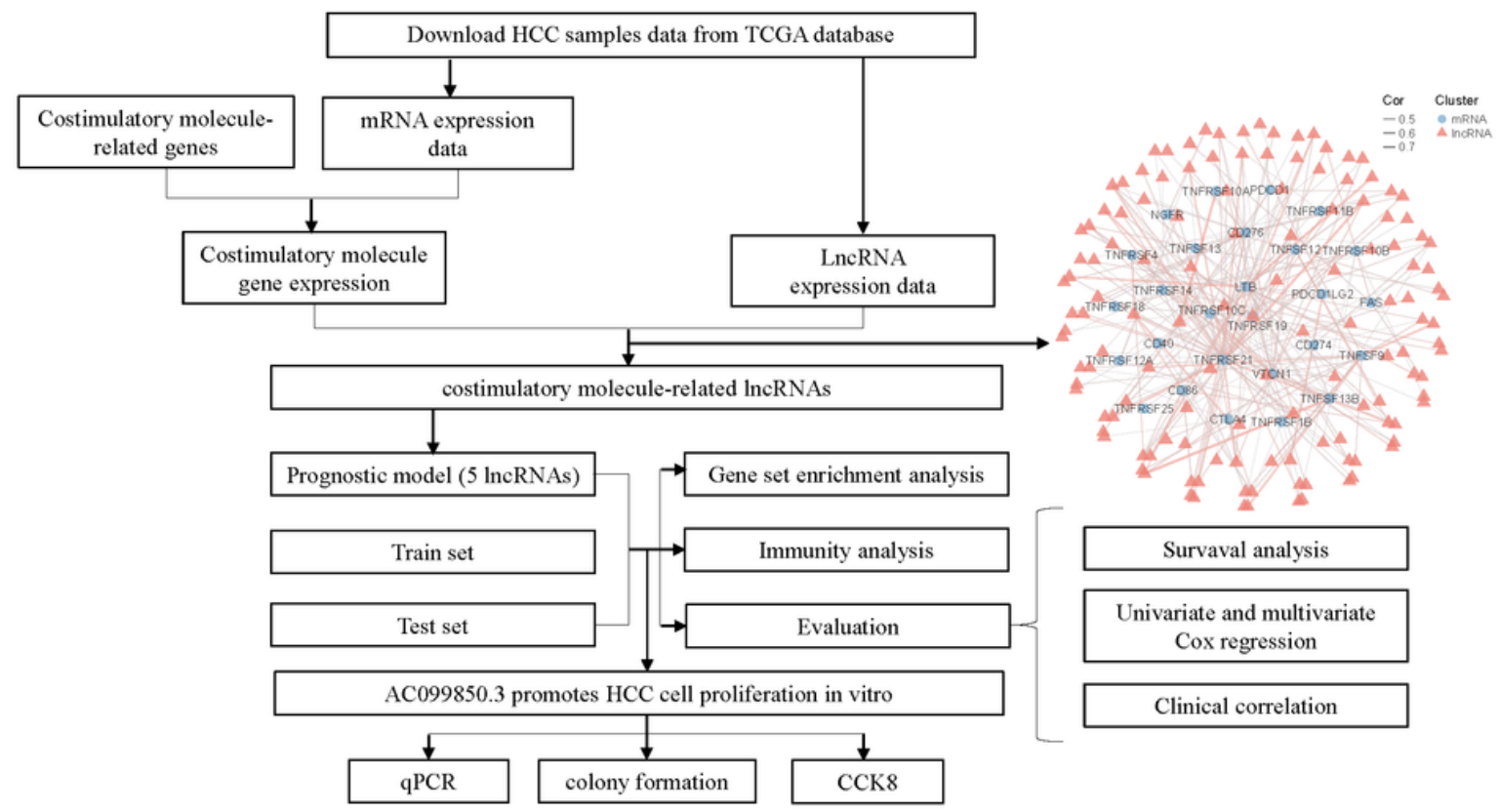

Figure 1

Flow chart 
A

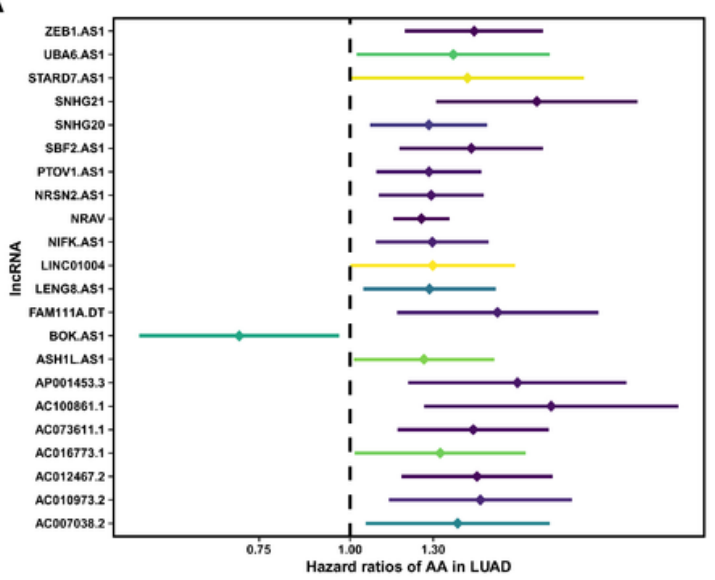

B

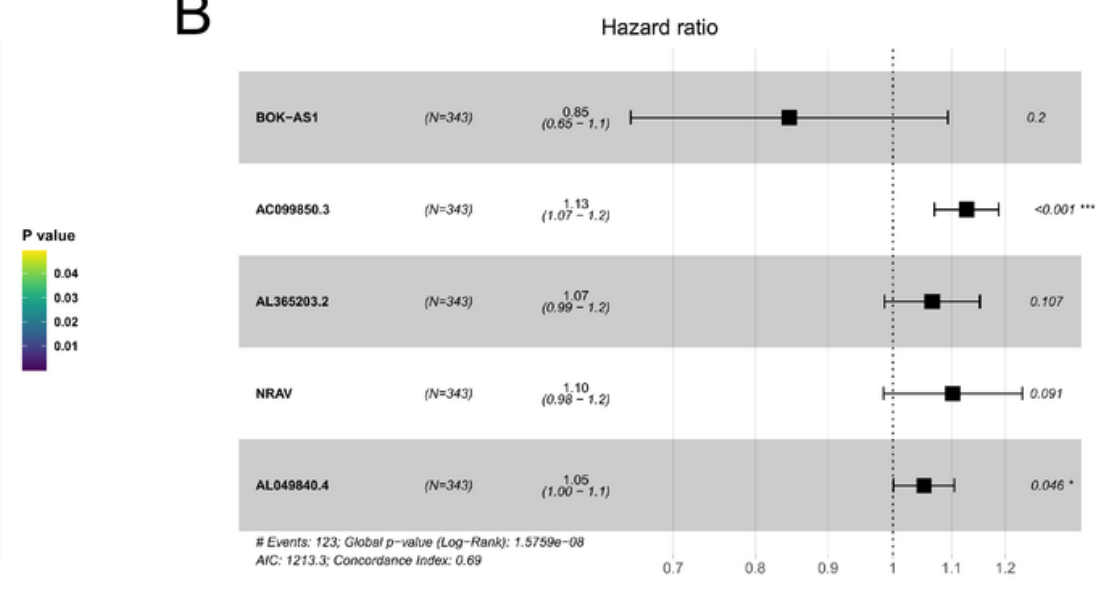

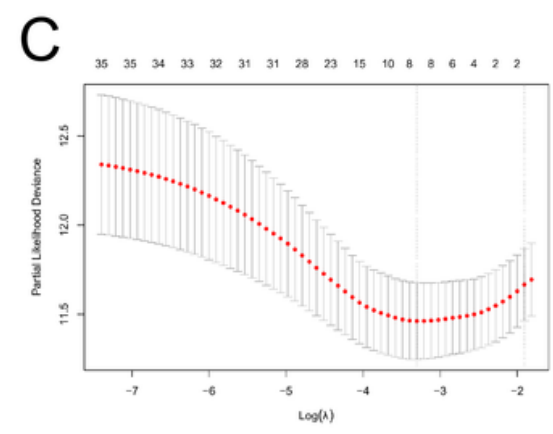
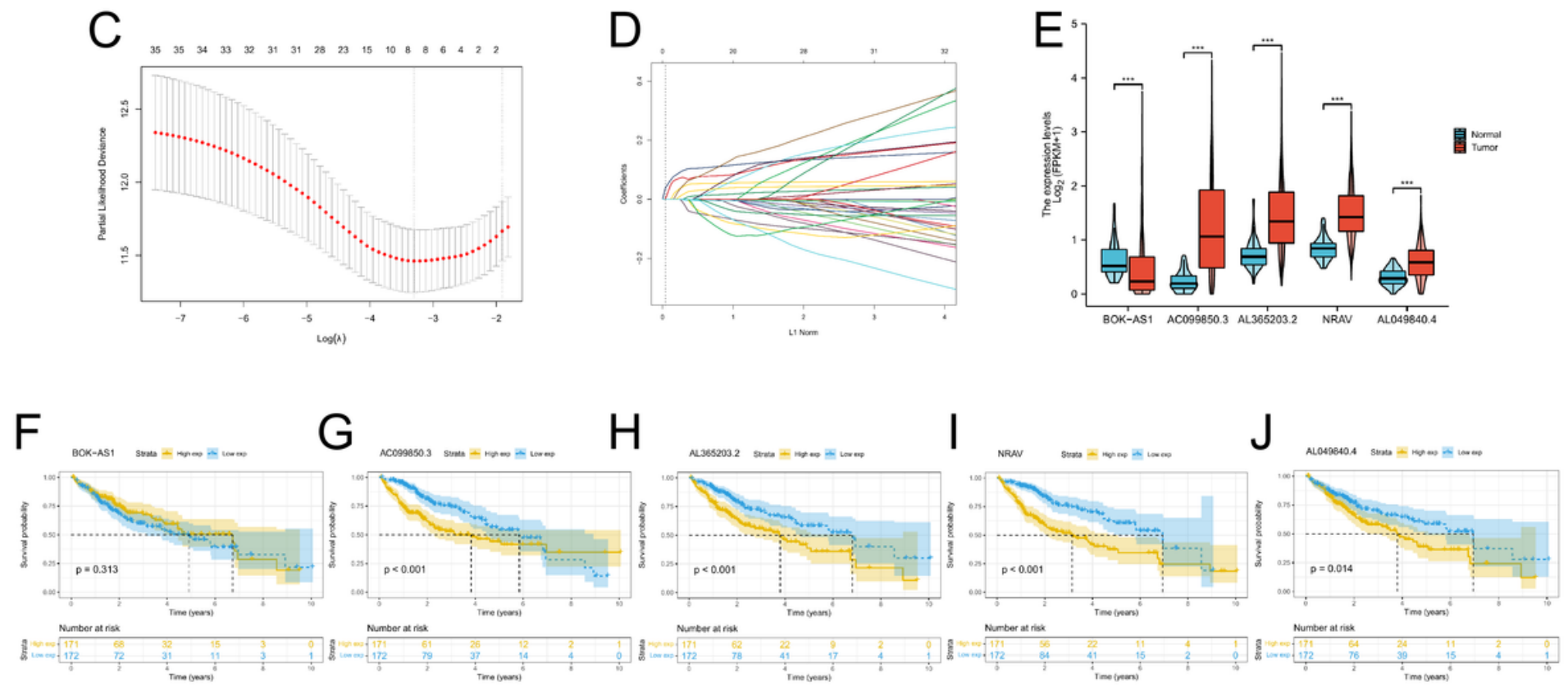

Figure 2

Identification of a prognostic molecule-related IncRNA signature. Notes: (A) Results of the univariate Cox regression analysis of prognostic costimulatory molecule-related IncRNAs in OS of HCC patients. (B) Results of the multivariate Cox regression analysis of prognostic molecule-related IncRNAs in OS of HCC patients. (C-D) LASSO regression of the costimulatory molecule-related IncRNAs selected from univariate Cox regression analysis. (E) Expression of five prognostic costimulatory molecule-related IncRNAs in HCC and normal tissue. (F-J) Kaplan-Meier survival curves of five prognostic costimulatory molecule-related IncRNAs in the HCC cohort. 

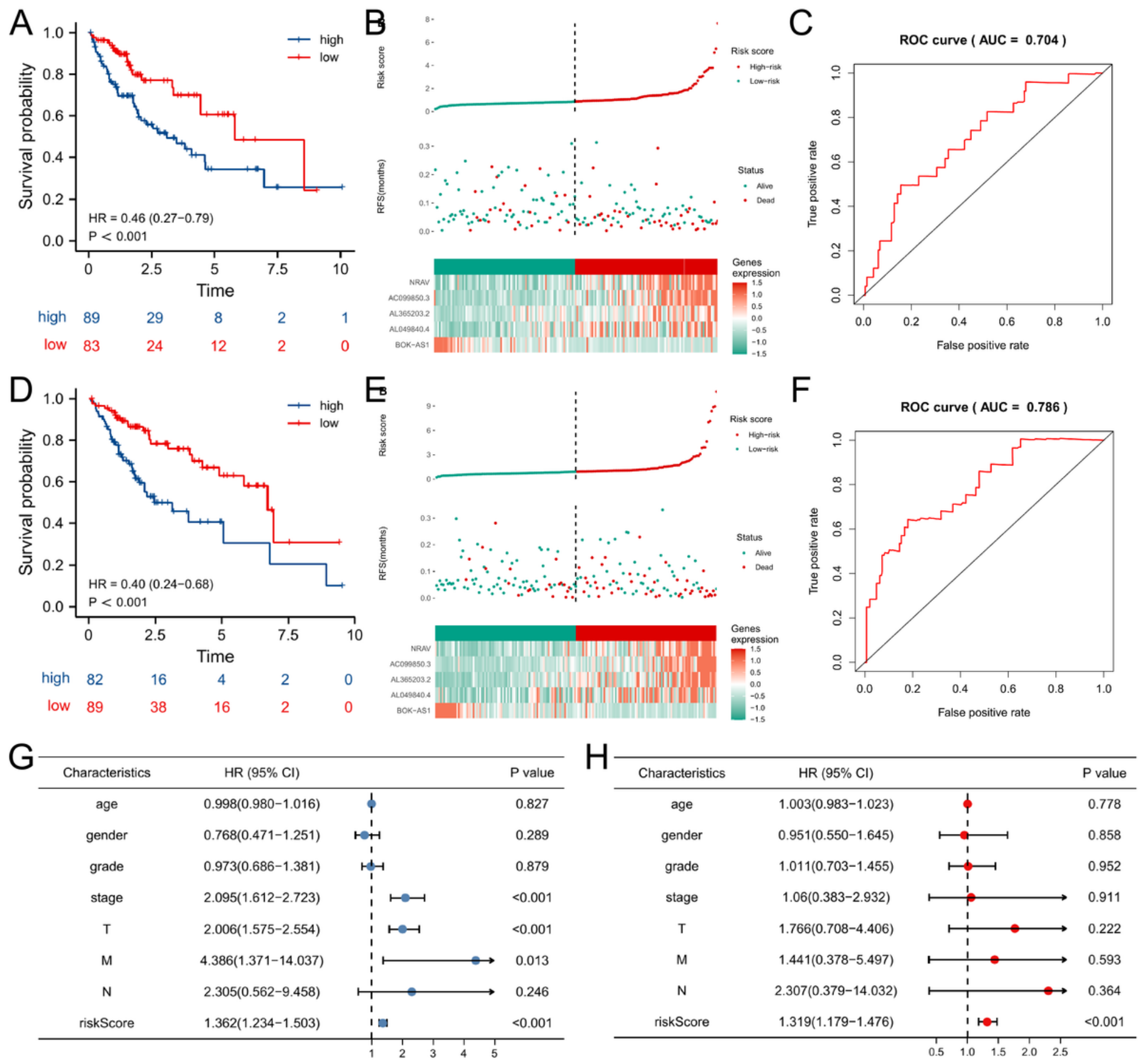

\section{Figure 3}

Evaluation and verification of the prognostic costimulatory molecule-related IncRNA signature. Notes: Kaplan-Meier survival curves for high- and low-risk patients in training (A) and testing cohort (D). The distribution and survival status of HCC patients with different risk scores. Respectively, the heatmap exhibited the expression levels of five prognostic costimulatory molecule-related IncRNAs in the high- and low-risk groups in training (B) and testing (E) cohort. The ROC curves of the prognostic signature in training $(\mathrm{C})$ and testing cohort $(\mathrm{F}) .(\mathrm{G}, \mathrm{H})$ Univariate and multivariate Cox regression analyses for the risk score as an independent prognostic factor. 

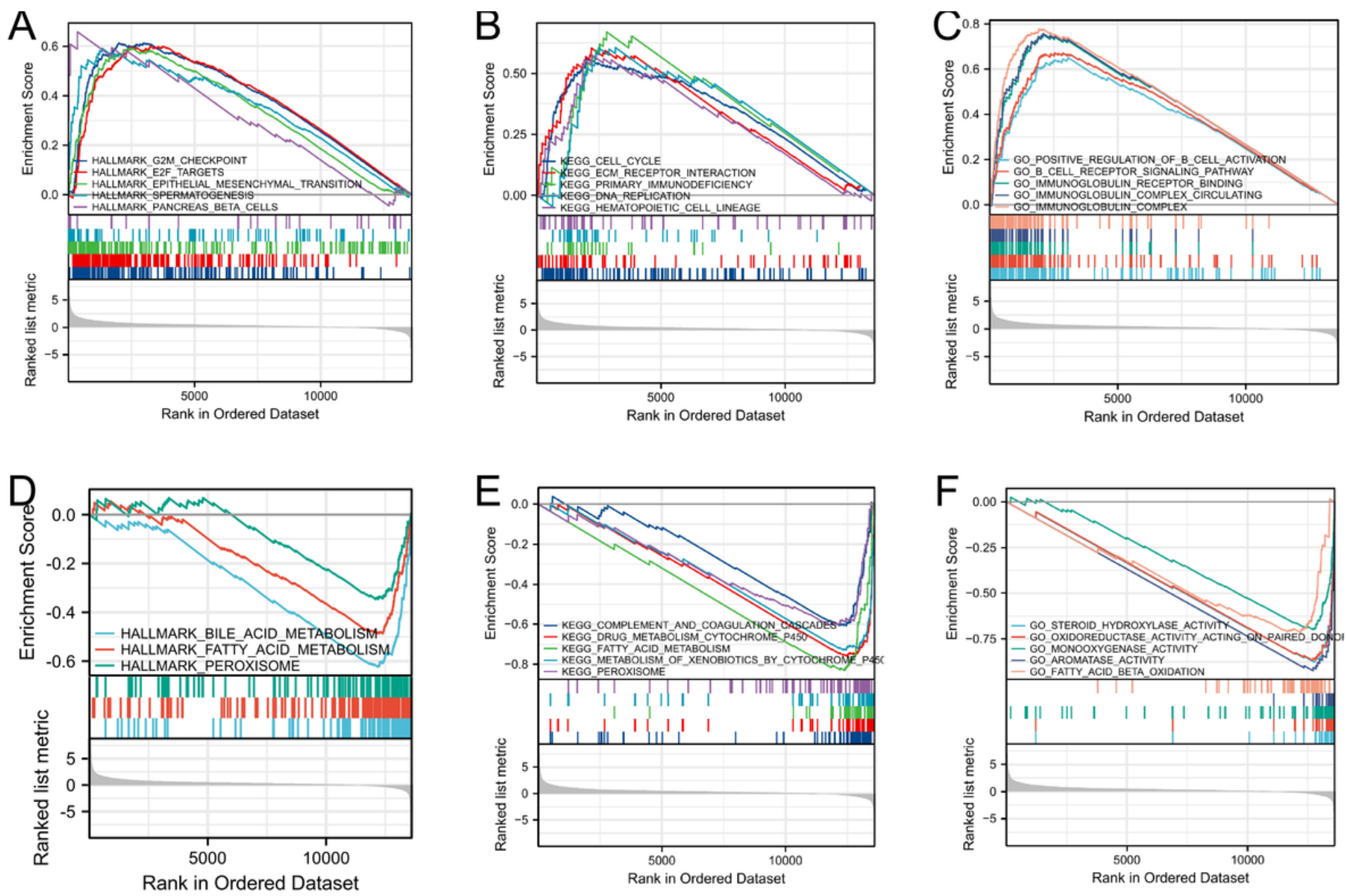

Figure 4

Gene set enrichment analysis for costimulatory molecule-related IncRNA signature. Notes: The top ten enriched gene sets are shown from gene set enrichment analysis of the costimulatory molecule-related IncRNAs biomarkers performed between the high- and low-risk groups using gene sets of HALLMARK $(\mathrm{A}, \mathrm{D}) \mathrm{KEGG}(\mathrm{B}, \mathrm{E})$ and $\mathrm{GO}(\mathrm{C}, \mathrm{F})$. 
A
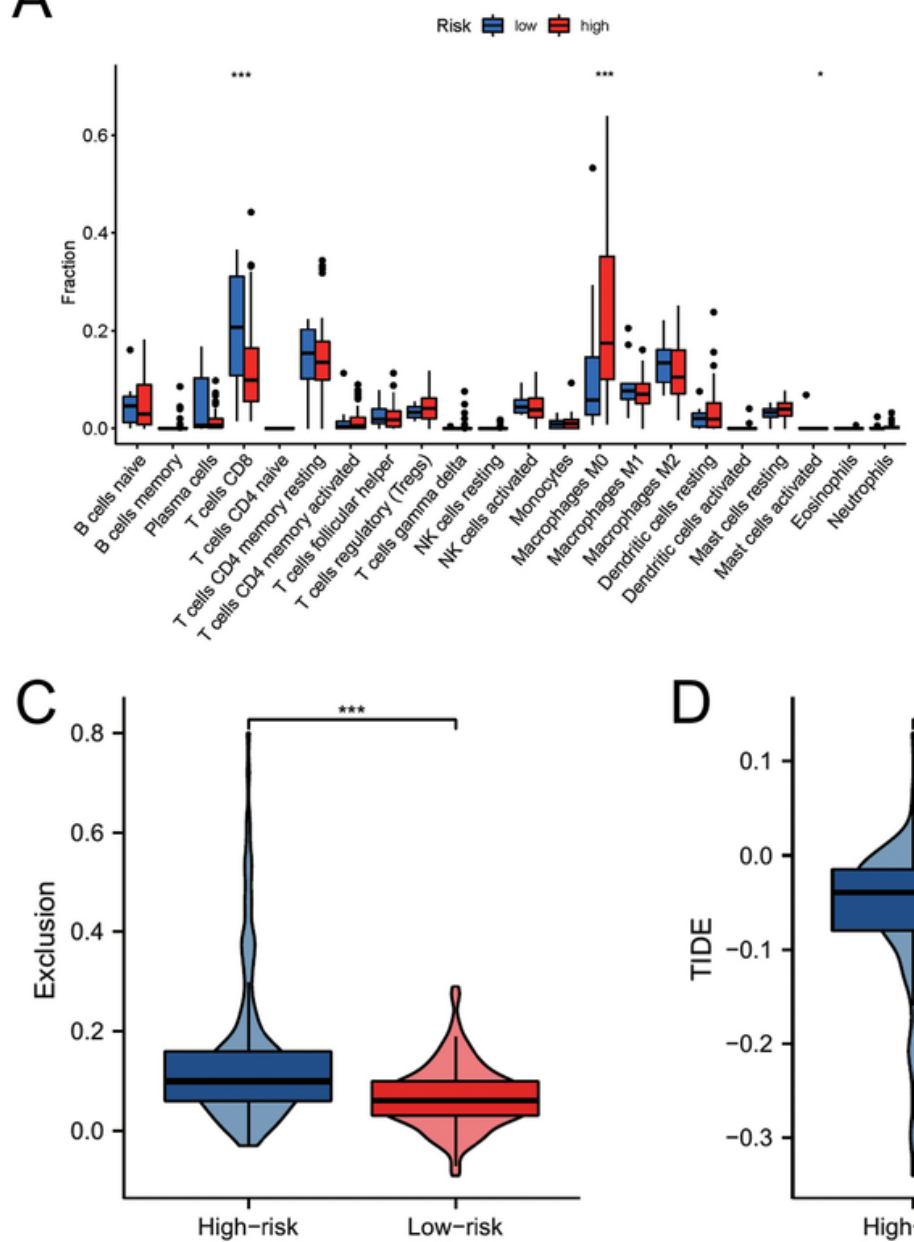

B
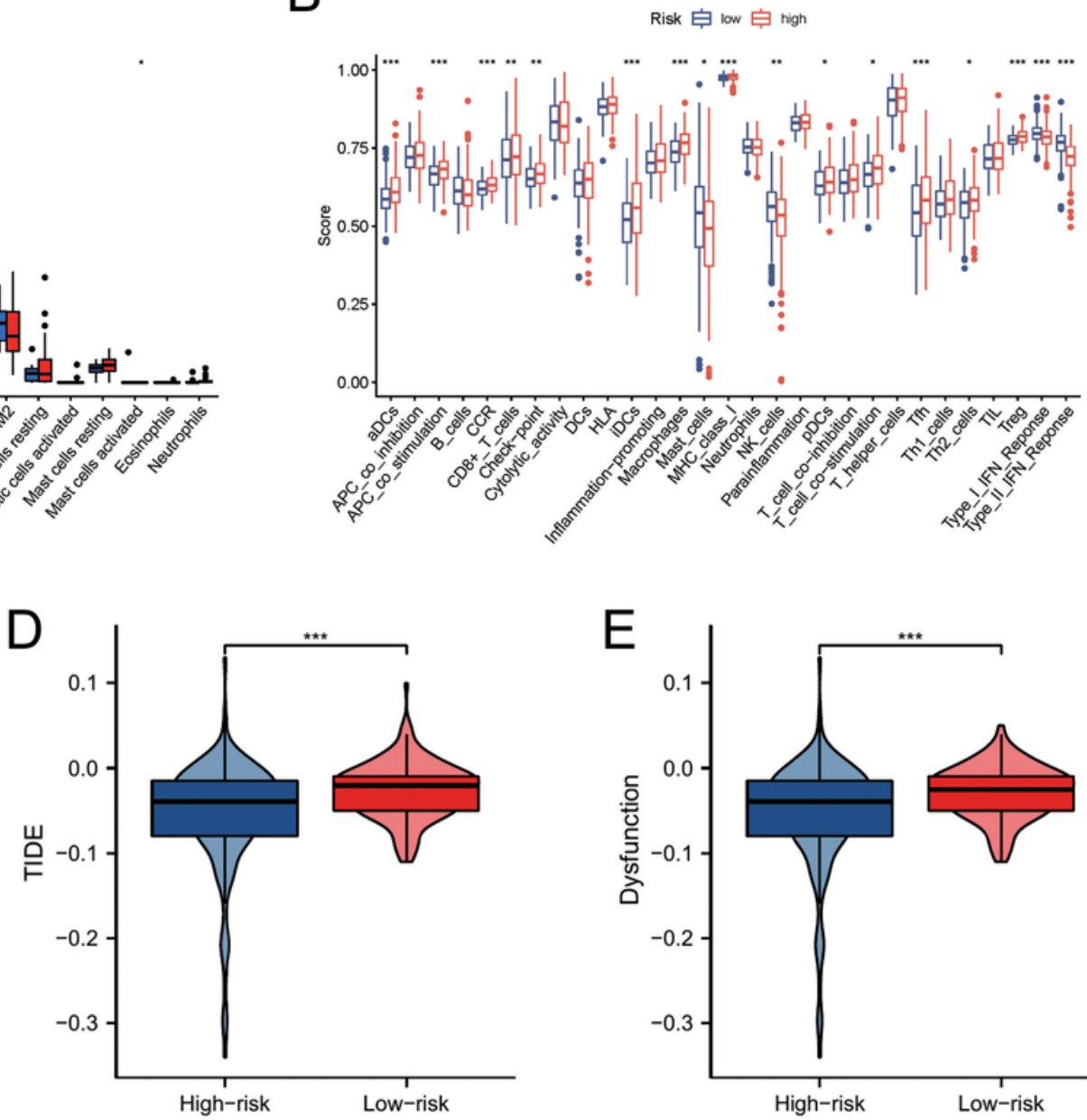

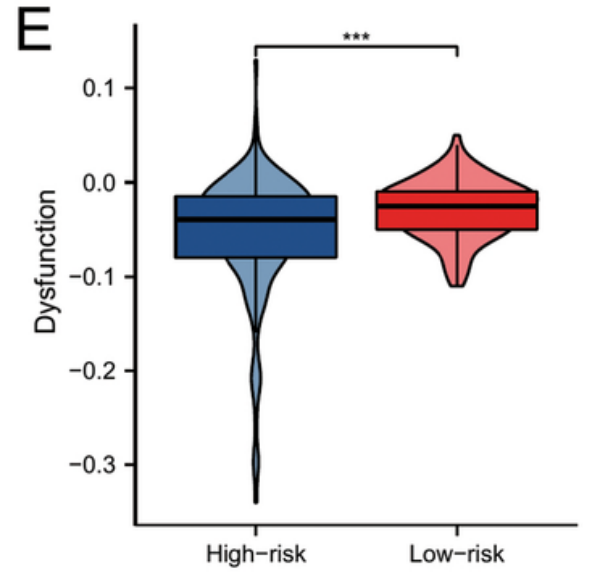

Figure 5

Patients with high- and low-risk scores had different immune statuses. Notes: Comparison of the ssGSEA scores of 22 types of immune cells (A) and 29 immune-related pathways (B) between low- and high-risk groups. (C) The high-risk group presented with a tendency of increased immune exclusion score value. Comparisons of TIDE (D) and immune dysfunction (E) score for chemotherapeutics and targeted therapy between two risk stratifications revealed that high-risk patients were more likely be suitable for immunotherapy. 

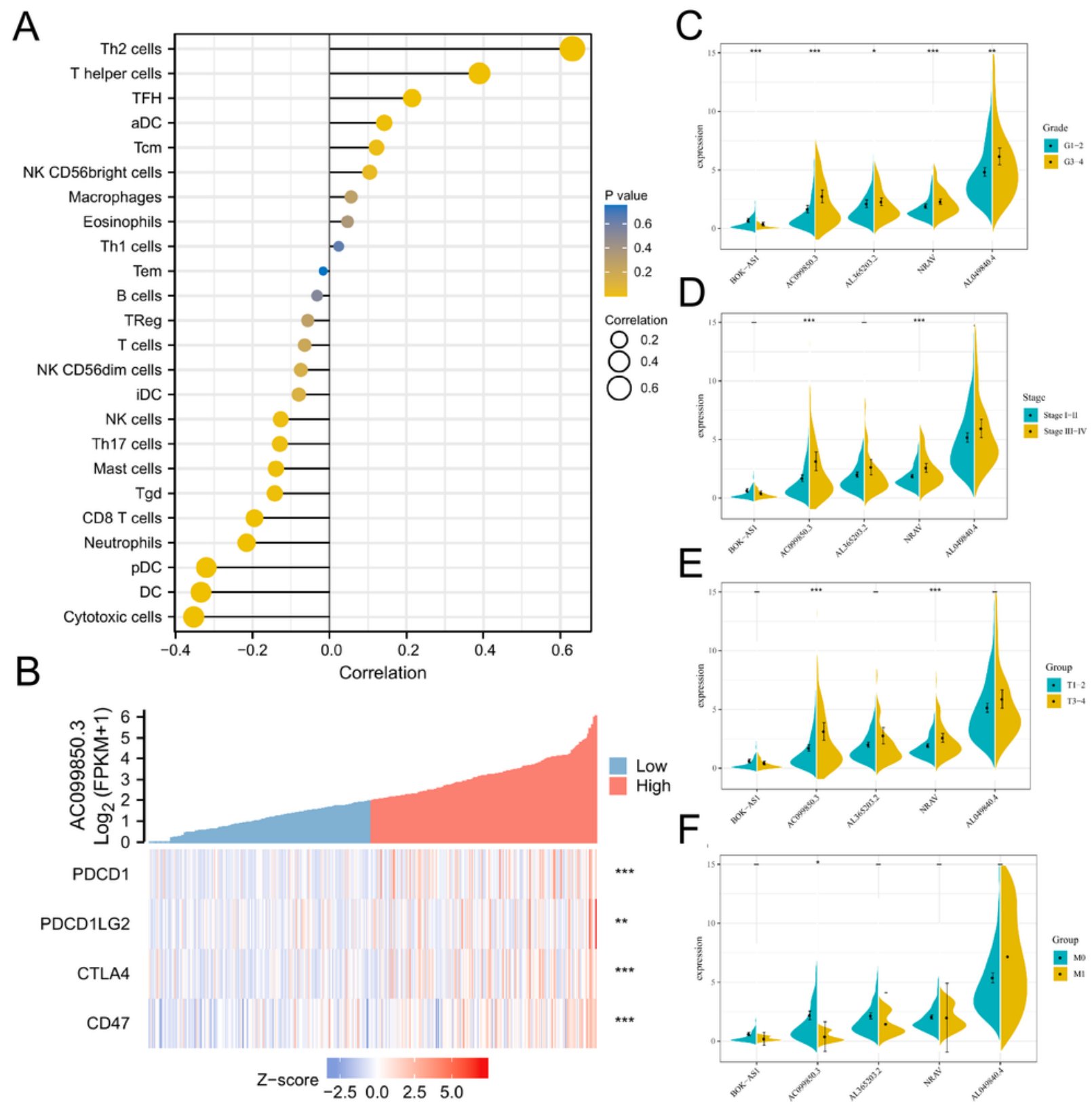

Figure 6

Clinical correlation analysis and the immunity features of AC099850.3 Notes: (A) Heat map showed the positive relations between the expression of AC099850.3 and several immune checkpoints. (B) Lollipopdiagram revealed that AC099850.3 have a strong correlation with some immune cells including Th2 cells and T helper cells. Violin plot showed that the AC099850.3 was more likely to be related with the progress of HCC, including Grade (C), Stage (D), T stage (E) and M stage (F) than other prognostic costimulatory molecule-related IncRNAs. 

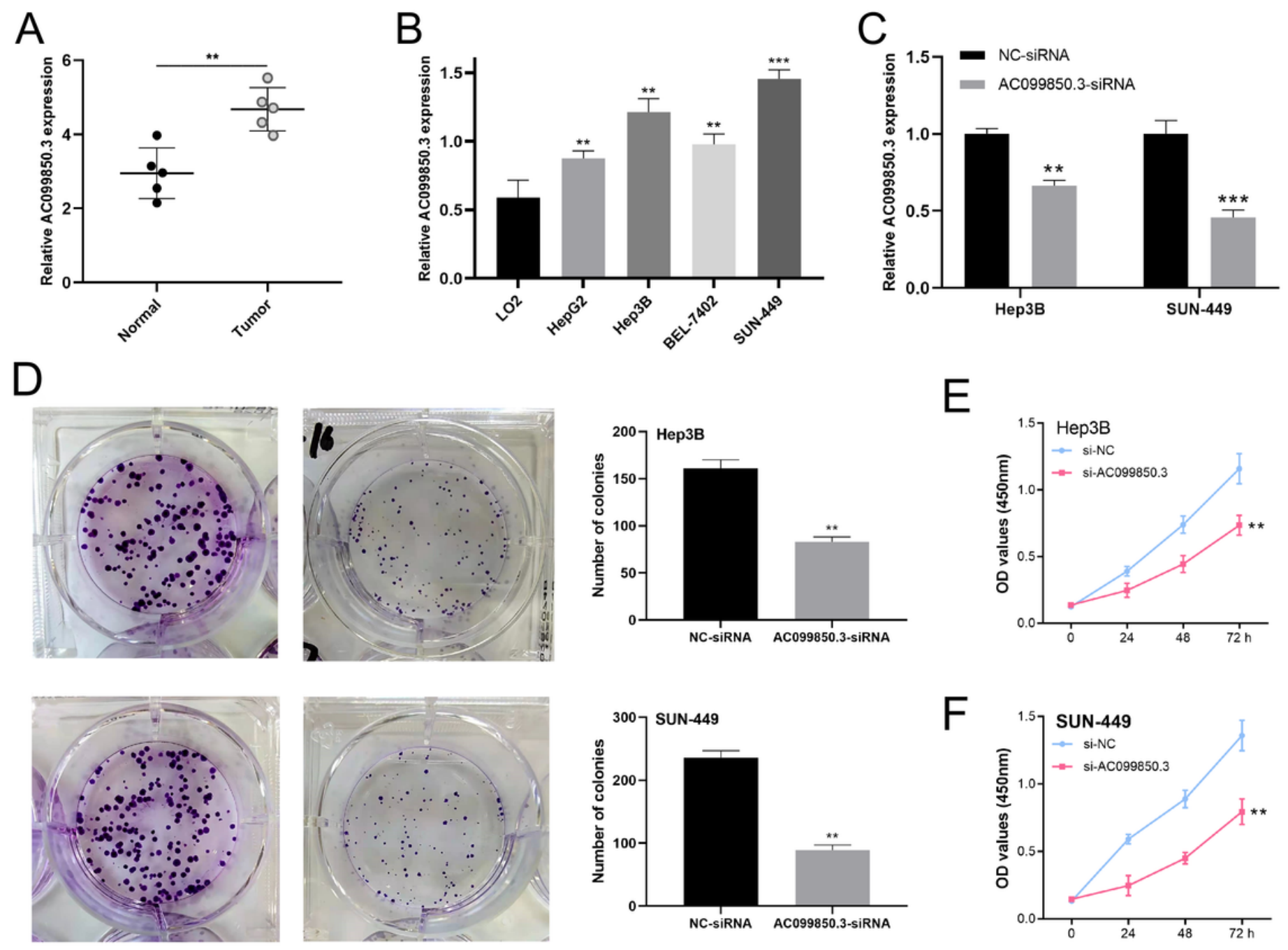

\section{Figure 7}

The expression levels of AC099850.3 and it promotes HCC cell proliferation in vitro. Notes: (A) AC099850.3 mRNA expression in tumors and non-malignant tissues from HCC patients. (B) AC099850.3 mRNA expression in HCC cell lines and normal hepatic cells. (C) mRNA expression in AC099850.3 knockdown cell lines. (D) Colony formation assay of Hep3B and SUN-449 after the knockdown of AC099850.3. CCK8 assay of Hep3B (E) and SUN-449 (F) after the knockdown of AC010973.2.

\section{Supplementary Files}

This is a list of supplementary files associated with this preprint. Click to download.

- Tables1.xls

- Tables2.xls

- Table2.docx 